\title{
The cornerstone of diagnostics and the treatment of psychotic states
}

\section{Editorial}

The target of the hard work of psychiatry specialists is to make a proper decision in the treatment of acute states in the psychiatry practise. That decision leads to the making of a better treatment protocol and a fast elimination of the symptoms of pychosis in order to prevent a decline in functioning and to establish the long term unressponsive symptoms of a disease. Also, not to lose the quality of life after the beggining of the psychosis.

A lot of papers and studies all over the world have brought new data about medication, the cost and benefits of different drugs and their advantages over each other.

I can rarely read about the details of diagnostics in clinical practice's. There are possible mistakes made when we are partly right and the therapeutic protocol might not be the best for the patient. Because of that I want to say something about the concrete situation, discutible symptoms and dilema's in the therapeutic aproach.

30 years ago, as a student on my last year of medicine in the University of Zagreb I was faced with an evident situation where a psychiatrist in private practice treated a psychotic state with an unspecific light antipsychotic. In the whole conversation there was no specific question that would differentiate most common diagnosis's from one another. The whole atmosphere and impression lead to my conclusion that the patient is schyzophrenic, and the family thought the same.

Ten years after that I became a specialist of psychiatry, and from then forward I have been working at the acute department in Šibenik, Croatia. The continuous experience of group psychotherapy and an analysis of the neurotic and psychotic states of the people was mixed with my everyday experience in hospital and my inpatient and outpatiet work with psychosis.

In my everyday practice, but also in the case documentation in papers and lectures I often notice that the treatment of psychotic states is not connected with a clear border between schizophrenia and bipolar disorder and usually, the patient and the family have are under the impression that the patient is diagnosed as schizophrenic.

Some of the bizzare symptoms seduce us and push us to think that it is schizophrenia and if we don't ask questions from the Jung mania rating scale to exclude BAP, than we can easily go toward the conclusion that it is a case of schizophrenia. When we are met with bizzare religious delusions we can easily miss the ideas of gloriness which lead to BAP. Symptoms of a depressive faze can flood the content of the interview and we may miss asking questions about the manic faze, especially the one's of a subjective sense of energy, need for sleep, fast talking, jumping from and to topics, spending more money than usual, great plans and unusual ambitiousness.

Another great decoy that turns towards the diagnostic opinion about a patient to schizophrenia is a change in the form of thinking. Faster fluency of thinking with fast changes of topics is often
Volume 3 Issue I - 2017

\author{
Milivoj Stojanovic \\ General Hospital Šibenik, Croatia
}

Correspondence: Milivoj Stojanovic, psychiatrist, General Hospital A ibenik, Croatia, Stjepana RadiAła 83, 22000 A ibenik Croatia, Tel +38598792485, Email milivoj.stojanovic7@gmail.com

Received: October 26, 2016 | Published: February 07, 2017

recognized as dissociative thinking. That assessment easily leads to the usage of antipsychotic treatment without a mood stabilizator. A lot of clinicians overrespect the possible adversive effects of lithium and avoid a treatment which is yet the golden standard for the treatment of BAP.

Some bizzare paranoiac delusions can be in the faze of mania which is a usual part of bipolar disorder. So, it is important not to recognize a lot of disphoric and hostile states in mania as paranoiac thinking and as a fundamental sign of schizophrenia.

Everything I say about diferential diagnosis sounds simple but it is incredibly easy to miss the right diagnose independently, even if you are an experienced clinician, including myself.

And if we recognize BAP, the best we can do for our patient? First of all, we don't unnecessarily stigmatize the patient with BAP with a diagnosis of schizoprenia. We motivate our patient to be an active part of the therapeutic union, the union between the patient and his psychiatrist who has the most important job, making the athmosfere one of a winner.

Early introduction of lithium at the beggining of the disease because of the process of maturation and provocation of neruronic growth in some regions of the brain can change the prognosis and a number of mood shifts in the future, also as a severity of mood disturbances. If the person with BAP is early and well stabilized and recognized on time, a lot of us in this field will know how good that person can function in familly and other interests.

In my practice, one crucial case report showed this problem in real life and sent arrows towards it being the possible subject of research in the future.

I have a patient in long term treatment of BAP. His daughter was about 17 years ago, a student of medicine, first year, in a university in western Europe. Her psychic state got worse at one moment in a caffe, she made a mess and said that she is the mother of Jesus. Quickly she was brought to a well known Clinic and recognised as F23.2 Acute schizofrenia like state. She was very inhibited with therapy and her parents took her from the Clinic to a private practice. Therapy has not much changed since that time, the initial doses of clozapine, sulpiride and promazine. After about three months she was admitted to our 
hospital. She looked very stiff and seemed to be blind, psychotic and desoriented. She lost it at some point in the summer. I removed all therapy and introduced lithium and haloperidole and after that I changed haloperidole with clozapine. Her state was bad, I suggested ECT therapy in anesthesia and we did six aplications three times a week.

After 10 days of treatment, stiffness was resolved and the psychotic state was over, as it hadn't ever existed. She was sent home from hospital after one month of treatment and was taking $75 \mathrm{mg}$ of clozapine per day. She took the medication only for 3-4 months and then stopped. After that, more than seventeen years of not taking any pharmacological treatment, she finished university and became a doctor of medicine. She graduated as a specialist in one field of medicine and at the moment works in her own private clinic. After 4-5 years of complete remission and after the results of some other cases I conclude that the neuronic growth provoked by ECT is responsible for the, possibly, lifetime recovery. Because of the resistance on every possible level I'll never make a study to compare the usual treatment of the first faze of BAP in the beginning of a disease with the treatment with ECT protocol together with medication lasts about 6-12 months.

One american study started seven years ago and was finished two years ago. They found out that that is the best protocol in the treatment of BAP. The chief of my staff, high medical nurse and some colleagues saw it on TV, but I haven't been able to find the autor. I called the TV editor but nobody could remember and I gave up.

At the end of this short story about real psychiatry and care for our patients I hope that my experience will be helpfull for usual practice.

\section{Acknowledgements}

None.

\section{Conflict of interest}

The auhtor declares no conflict of interest. 